Georgian Mathematical Journal

Volume 11 (2004), Number 3, 479-487

\title{
ON NEGLIGIBLE AND ABSOLUTELY NONMEASURABLE SUBSETS OF THE EUCLIDEAN PLANE
}

\author{
A. KHARAZISHVILI
}

\begin{abstract}
The notions of a negligible set and of an absolutely nonmeasurable set are introduced and discussed in connection with the measure extension problem. In particular, it is demonstrated that there exist subsets of the plane $\mathbf{R}^{2}$ which are $T_{2}$-negligible and, simultaneously, $G$-absolutely nonmeasurable. Here $T_{2}$ denotes the group of all translations of $\mathbf{R}^{2}$ and $G$ denotes the group generated by $\{g\} \cup T_{2}$, where $g$ is an arbitrary rotation of $\mathbf{R}^{2}$ distinct from the identity transformation and all central symmetries of $\mathbf{R}^{2}$.
\end{abstract}

2000 Mathematics Subject Classification: 28A05, 28D05.

Key words and phrases: Sierpiński partition, quasi-invariant measure, uniform set, negligible set, absolutely nonmeasurable set.

It is well known that the group of all motions of the Euclidean plane $\mathbf{R}^{2}$ is solvable hence amenable and, in fact, there are no equidecomposability paradoxes in $\mathbf{R}^{2}$ (in this context, the classical Banach theorem on the existence of universal finitely additive motion-invariant normalized measures on $\mathbf{R}^{2}$ must be indicated). However, various subsets of $\mathbf{R}^{2}$ were constructed with strange and, in some sense, paradoxical geometric properties.

The best known example of such a set is due to Mazurkiewicz. Recall that he constructed a subset of the plane which meets every straight line of the plane in exactly two points.

Other interesting examples are due to Sierpiński and Davies (see [1] and [2]). Let us remind that in his famous work [1], Sierpiński showed that the Continuum Hypothesis is equivalent to the existence of a partition of the plane $\mathbf{R}^{2}$ into two sets $A$ and $B$, such that $A$ meets every straight line parallel to the axis $\mathbf{R} \times\{0\}$ in countably many points and, similarly, $B$ meets every straight line parallel to the axis $\{0\} \times \mathbf{R}$ in countably many points.

It should be underlined that this partition became a starting point for further investigations concerning plane sets with strange properties from the measuretheoretical viewpoint (see, for instance, [3]).

In the present article we are going to give one application of the abovementioned Sierpiński partition to constructions of sets which, on the one hand, are rather good with respect to translation-invariant measures on the plane $\mathbf{R}^{2}$ and, on the other hand, are extremely bad with respect to motion-invariant measures on $\mathbf{R}^{2}$. Moreover, applying the method developed in [4] (cf. also [5]), we are able to extend constructions of this sort to the case of the Euclidean space $\mathbf{R}^{n}$ where $n \geq 3$.

Throughout this article, it will be convenient to utilize the following notation: 
$T_{n}=$ the group of all translations of the space $\mathbf{R}^{n}$;

$S_{n}=$ the group generated by all central symmetries of $\mathbf{R}^{n}$;

$M_{n}=$ the group of all motions (i.e., isometric transformations) of $\mathbf{R}^{n}$.

Note that, for $n \geq 2$, we have the following proper inclusions:

$$
T_{n} \subset S_{n} \subset M_{n}
$$

Note also that the group $S_{n}$ is generated by $T_{n}$ and the symmetry of the space $\mathbf{R}^{n}$ with respect to its origin. Moreover, $T_{n}$ is a normal subgroup of $M_{n}$.

We also recall that the symbol $O_{n}$ denotes the group of all linear orthogonal transformations of $\mathbf{R}^{n}$. Respectively, the symbol $O_{n}^{+}$stands for the group of all proper orthogonal transformations of $\mathbf{R}^{n}$ which are usually called the rotations of this space (about its origin).

If $n=1$, then $O_{n}$ consists exactly of two elements: the identity transformation and the symmetry with respect to the origin. Therefore, $O_{n}^{+}$is trivial, i.e., is reduced to the identity transformation.

If $n=2$, then $O_{n}$ consists of all rotations (about the origin) and all symmetries with respect to straight lines passing through the origin. At the same time, the group $O_{n}^{+}$is commutative and is canonically isomorphic to the unit circumference $\mathbf{S}_{1}$.

For $n \geq 3$, the algebraic structure of $O_{n}^{+}$becomes more complicated. Namely, it turns out that this group contains a free subgroup of cardinality continuum. This fact was first established by Sierpiński (see [6]) and it implies the existence of various strong equidecomposability paradoxes in $\mathbf{R}^{n}$ or, respectively, in $\mathbf{S}_{n-1}$ (for details, see [7]).

As a rule, paradoxical subsets of $\mathbf{R}^{n}$ turn out to be very bad from the measuretheoretical viewpoint.

Let us introduce some definitions from the theory of invariant (more generally, quasi-invariant) measures (cf. [4] and [5]).

Let $E$ be a nonempty set and let $G$ be a fixed group of transformations of $E$.

Let $X$ be a subset of $E$. We shall say that $X$ is $G$-negligible (in $E$ ) if the following two conditions are satisfied:

(a) there exists at least one nonzero $\sigma$-finite $G$-quasi-invariant measure $\mu$ on $E$ such that $X \in \operatorname{dom}(\mu)$ and $\mu(X)=0$;

(b) for any nonzero $\sigma$-finite $G$-quasi-invariant measure $\nu$ on $E$, the relation $X \in \operatorname{dom}(\nu)$ implies $\nu(X)=0$.

Let $Y$ be a subset of $E$. We shall say that $Y$ is $G$-absolutely nonmeasurable (in $E$ ) if there exists no nonzero $\sigma$-finite $G$-quasi-invariant measure $\nu$ on $E$ such that $Y \in \operatorname{dom}(\nu)$.

Some properties of negligible and absolutely nonmeasurable sets are discussed in [4] and [5]. Here we focus our attention on analogous sets in the Euclidean plane $\mathbf{R}^{2}$.

Let $X$ be a subset of $\mathbf{R}^{2}$. Recall that $X$ is said to be uniform in $\mathbf{R}^{2}$ if there exists a straight line $p \subset \mathbf{R}^{2}$ such that $\operatorname{card}\left(p^{\prime} \cap X\right) \leq 1$ for every line $p^{\prime}$ parallel to $p$. In this case $X$ is also said to be uniform with respect to $p$. 
Example 1. If $X$ is a uniform subset of $\mathbf{R}^{2}$, then $X$ is $T_{2}$-negligible (and $S_{2}$-negligible). Moreover, it was shown in [8] that if $p$ is a straight line in $\mathbf{R}^{2}$ and a set $X \subset \mathbf{R}^{2}$ is such that every line parallel to $p$ meets $X$ in finitely many points, then $X$ is $T_{2}$-negligible (and $S_{2}$-negligible). Actually, a similar result can be formulated and proved for sets which are finite with respect to an uncountable subgroup of a given commutative group (see [9]). In particular, if every line parallel to $p$ meets $X$ in one or two points, then $X$ turns out to be $T_{2}$-negligible $\left(S_{2}\right.$-negligible). This fact will be employed below.

Example 2. Assuming the Continuum Hypothesis and starting with a Sierpiński partition of $\mathbf{R}^{2}$, it is not hard to define two sets $A^{\prime}$ and $B^{\prime}$ in $\mathbf{R}^{2}$ such that:

(1) the set $A^{\prime}$ is uniform with respect to the axis $\mathbf{R} \times\{0\}$;

(2) the set $B^{\prime}$ is uniform with respect to the axis $\{0\} \times \mathbf{R}$;

(3) there exists a countable family $\left\{h_{n}: n<\omega\right\}$ of translations of $\mathbf{R}^{2}$, for which we have

$$
\cup\left\{h_{n}\left(A^{\prime} \cup B^{\prime}\right): n<\omega\right\}=\mathbf{R}^{2} .
$$

Conversely, the existence of sets $A^{\prime}$ and $B^{\prime}$ satisfying the properties (1)-(3) implies the validity of the Continuum Hypothesis. Note also that these properties of $A^{\prime}$ and $B^{\prime}$ enable us to demonstrate the $T_{2}$-absolute nonmeasurability (hence, $S_{2}$-absolute nonmeasurability and $M_{2}$-absolute nonmeasurability) of the set $A^{\prime} \cup B^{\prime}$. Indeed, let $\nu$ be an arbitrary nonzero $\sigma$-finite $T_{2}$-quasi-invariant measure on $\mathbf{R}^{2}$ and suppose for a while that

$$
A^{\prime} \cup B^{\prime} \in \operatorname{dom}(\nu) \text {. }
$$

Without loss of generality, we may assume that $\nu$ is a probability measure. Now, relation (3) shows that

$$
\nu\left(A^{\prime} \cup B^{\prime}\right)>0 .
$$

On the other hand, by applying the classical Banach theorem (see, e.g., [4] or $[7]$ ), we can extend $\nu$ to a finitely additive $T_{2}$-quasi-invariant measure $\nu^{\prime}$ defined on the family of all subsets of $\mathbf{R}^{2}$. Since both the sets $A^{\prime}$ and $B^{\prime}$ are uniform, we get

$$
\nu^{\prime}\left(A^{\prime}\right)=\nu^{\prime}\left(B^{\prime}\right)=0
$$

On the other hand, we have

$$
0<\nu\left(A^{\prime} \cup B^{\prime}\right)=\nu^{\prime}\left(A^{\prime} \cup B^{\prime}\right) \leq \nu^{\prime}\left(A^{\prime}\right)+\nu^{\prime}\left(B^{\prime}\right)=0,
$$

which yields a contradiction. The contradiction obtained establishes the $T_{2^{-}}$ absolute nonmeasurability of $A^{\prime} \cup B^{\prime}$.

Now, let $g$ denote the rotation of the plane $\mathbf{R}^{2}$ (about its origin), which maps the axis $\mathbf{R} \times\{0\}$ onto the axis $\{0\} \times \mathbf{R}$. Let us consider the set

$$
Z=g\left(A^{\prime}\right) \cup B^{\prime} \text {. }
$$

Since every straight line lying in $\mathbf{R}^{2}$ and parallel to the axis $\{0\} \times \mathbf{R}$ meets the set $Z$ in one or two points, we may assert that $Z$ is $T_{2}$-negligible in $\mathbf{R}^{2}$ (cf. 
Example 1). At the same time, it can easily be observed that $Z$ is $G$-absolutely nonmeasurable in $\mathbf{R}^{2}$, where

$$
G=\left[\{g\} \cup T_{2}\right]
$$

denotes the group generated by $g$ and $T_{2}$. To see this, let us take a probability $G$-quasi-invariant measure $\nu$ on $\mathbf{R}^{2}$ and suppose for a moment that $Z \in \operatorname{dom}(\nu)$. Without loss of generality, we may assume that $\nu$ is complete. Since $T_{2} \subset G$, the measure $\nu$ is also $T_{2}$-quasi-invariant and we must have $\nu(Z)=0$ (in view of the $T_{2}$-negligibility of $Z$ ). Therefore, $\nu\left(g^{-1}(Z)\right)=0$ by virtue of the $G$-quasiinvariance of $\nu$. Hence, the equalities

$$
\nu\left(A^{\prime}\right)=0, \quad \nu\left(B^{\prime}\right)=0
$$

are valid and yield $\nu\left(A^{\prime} \cup B^{\prime}\right)=0$. Now, applying (3), we easily come to the equality $\nu\left(\mathbf{R}^{2}\right)=0$ which contradicts our assumption that $\nu\left(\mathbf{R}^{2}\right)=1$. The contradiction obtained gives the required statement. Note that a slight modification of this argument leads to a more general result stating (under $\mathbf{C H}$ ) that there exists a uniform subset $X$ of $\mathbf{R}^{2}$ which covers $\mathbf{R}^{2}$ with the aid of countably many transformations from $G$, i.e.,

$$
\cup\left\{g_{k}(X): k<\omega\right\}=\mathbf{R}^{2}
$$

for some countable family $\left\{g_{k}: k<\omega\right\} \subset G$. In particular, $X$ is $T_{2}$-negligible and $G$-absolutely nonmeasurable.

Example 3. Let $\left\{p_{k}: k<\omega\right\}$ be an injective countable family of straight lines in $\mathbf{R}^{2}$ passing through the origin. Recall the result of Davies who has demonstrated in [2] that there exists a family $\left\{X_{k}: k<\omega\right\}$ of subsets of $\mathbf{R}^{2}$ satisfying the following conditions:

(1) $\cup\left\{X_{k}: k<\omega\right\}=\mathbf{R}^{2}$;

(2) for each $k<\omega$, the set $X_{k}$ is uniform with respect to the line $p_{k}$.

It is not hard to deduce from this result that there exists a uniform subset $Z$ of $\mathbf{R}^{2}$ such that

$$
\cup\left\{f_{i}(Z): i<\omega\right\}=\mathbf{R}^{2}
$$

for some countable family $\left\{f_{i}: i<\omega\right\}$ of motions of the plane. In particular, the set $Z$ turns out to be $T_{2}$-negligible and $M_{2}$-absolutely nonmeasurable.

In the sequel, we need several auxiliary propositions.

Lemma 1. Let $g$ be a rotation of the plane $\mathbf{R}^{2}$ (about its origin 0 ). Then there exists a field $P \subset \mathbf{R}$ of cardinality $\omega_{1}$, such that $\mathbf{R}^{2}$ can be represented in the form

$$
\mathbf{R}^{2}=U+V \quad(U \cap V=\{0\}),
$$

where $U$ and $V$ satisfy the following conditions:

1) $U$ is a two-dimensional vector subspace of $\mathbf{R}^{2}$ over $P$;

2) $g(U)=U$;

3) $V$ is a vector subspace of $\mathbf{R}^{2}$ over $P$. 
Proof. Let $\left\{e_{1}, e_{2}\right\}$ be the canonical orthonormal basis in $\mathbf{R}^{2}$. Obviously, we can write

$$
g\left(e_{1}\right)=a_{1} e_{1}+a_{2} e_{2}, \quad g\left(e_{2}\right)=b_{1} e_{1}+b_{2} e_{2},
$$

where $a_{1}, a_{2}, b_{1}, b_{2}$ are some (uniquely determined) real coefficients for which we have

$$
1=\left|a_{1} b_{2}-a_{2} b_{1}\right| \neq 0 .
$$

Let $P$ be a subfield of $\mathbf{R}$ of cardinality $\omega_{1}$, such that

$$
\left\{a_{1}, a_{2}, b_{1}, b_{2}\right\} \subset P .
$$

This subfield can readily be constructed (e.g., by the method of transfinite recursion). We put

$$
U=P e_{1}+P e_{2} .
$$

It is easy to verify that $g(U)=U$. Finally, take as $V$ an arbitrary vector space (over the same $P$ ) satisfying the relations

$$
U+V=\mathbf{R}^{2}, \quad U \cap V=\{0\} .
$$

The existence of such a $V$ is evident.

Lemma 2. Let $G_{1}$ and $G_{2}$ be any two groups identified, respectively, with the groups of their left translations, let

$$
\phi: G_{1} \rightarrow G_{2}
$$

be a surjective homomorphism and suppose that $X$ is a $G_{2}$-negligible subset of $G_{2}$. Then the set $\phi^{-1}(X)$ is $G_{1}$-negligible in $G_{1}$.

We omit an easy proof of Lemma 2.

Lemma 3. Let $E$ be a set, $G$ be a group of transformations of $E$ and let $Y$ be a $G$-absolutely nonmeasurable set in $E$. Then there exists a countable family $\left\{g_{k}: k<\omega\right\}$ of transformations from $G$, such that

$$
\cup\left\{g_{k}(Y): k<\omega\right\}=E \text {. }
$$

Proof. Suppose to the contrary that

$$
\cup\left\{g_{k}(Y): k<\omega\right\} \neq E
$$

for any countable family $\left\{g_{k}: k<\omega\right\} \subset G$. Then $Y$ is a member of some $G$-invariant $\sigma$-ideal $\mathcal{I}$ of subsets of $E$. Obviously, there exists a $G$-invariant probability measure $\mu$ on $E$ such that $\mathcal{I}=\mathcal{I}(\mu)$. In particular, $Y \in \operatorname{dom}(\mu)$. Hence, $Y$ cannot be $G$-absolutely nonmeasurable. The contradiction obtained finishes the proof.

Lemma 4. Let $E$ be a set, $G_{1}$ and $G_{2}$ be two groups of transformations of $E$, such that $G_{1} \subset G_{2}$, and let $Z$ be a $G_{1}$-negligible set in $E$. Suppose also that there exists a countable family $\left\{g_{i}: i<\omega\right\} \subset G_{2}$ for which

$$
\cup\left\{g_{i}(Z): i<\omega\right\}=E .
$$

Then the set $Z$ is $G_{2}$-absolutely nonmeasurable in $E$. 
The proof of this lemma is almost trivial and is omitted.

Now, we can formulate and prove the following statement.

Theorem 1. Let $g$ be a rotation of the plane $\mathbf{R}^{2}$, distinct from the identity transformation of $\mathbf{R}^{2}$ and all central symmetries of $\mathbf{R}^{2}$. Then there exists a set $Z \subset \mathbf{R}^{2}$ such that:

1) $Z$ is $T_{2}$-negligible (and $S_{2}$-negligible) in $\mathbf{R}^{2}$;

2) $Z$ is $G$-absolutely nonmeasurable in $\mathbf{R}^{2}$, where $G=\left[T_{2} \cup\{g\}\right]$.

Proof. The argument below is very similar to the one given in Example 2. First of all, we may assume without loss of generality that the fixed point of $g$ coincides with the origin 0 of $\mathbf{R}^{2}$, i.e., $g \in O_{2}^{+}$. Let us represent $\mathbf{R}^{2}$ in the form

$$
\mathbf{R}^{2}=U+V \quad(U \cap V=\{0\}),
$$

where $U$ and $V$ are vector spaces over the field $P \subset \mathbf{R}$ described in Lemma 1. For the two-dimensional vector space $U$, consider an analog of the Sierpiński partition, corresponding to the following two axes in $U$ : the "line" $P e_{1}$ and its image under the transformation $g$ (in fact, we are dealing here with a certain "affine" version of the Sierpiński partition for $U$ ). Now, it is not hard to verify that the argument of Example 2 works in our situation as well, and we do not need the Continuum Hypothesis since

$$
\operatorname{card}(U)=\operatorname{card}(P \times P)=\operatorname{card}(P)=\omega_{1} .
$$

Therefore, we obtain a set $X \subset U$ which is uniform in $U$ (with respect to the "line" $P e_{1}$ ) and has the property that the union $X \cup g(X)$ covers $U$ by using countably many translations of $U$. In particular, we see that $X$ is a $U$ negligible and $G^{\prime}$-absolutely nonmeasurable subset of $U$, where $G^{\prime}$ denotes the group generated by $U$ and $g \mid U$. Further, we define

$$
Z=(X+V) \cup\left(X+g^{-1}(V)\right) .
$$

We assert that $Z$ is the required set in $\mathbf{R}^{2}$. In order to demonstrate this, let us first observe that

$$
g^{-1}(V) \cap U=\{0\},
$$

whence it follows that every "line" in $\mathbf{R}^{2}$ parallel to $P e_{1}$ meets $Z$ in one or two points. This implies that $Z$ is a $T_{2}$-negligible subset of $\mathbf{R}^{2}$ (cf. Example 1 ). On the other hand, we have the inclusion

$$
(X \cup g(X))+V \subset Z \cup g(Z)
$$

which yields that the union $Z \cup g(Z)$ covers $\mathbf{R}^{2}$ with the aid of countably many translations of $\mathbf{R}^{2}$. So we can conclude, in view of Lemma 4 , that the set $Z$ is $G$-absolutely nonmeasurable in $\mathbf{R}^{2}$.

Remark 1 . Let $g$ be a rotation of $\mathbf{R}^{2}$ about its origin 0 . Suppose also that $g$ is of infinite order, i.e., $g^{k}$ differs from the identity transformation of $\mathbf{R}^{2}$ for all strictly positive integers $k$. Take any straight line $p_{0}$ in $\mathbf{R}^{2}$ passing through 0 and put

$$
p_{k}=g^{k}\left(p_{0}\right) \quad(k<\omega)
$$


We get the injective countable family of straight lines $p_{k}(k<\omega)$, to which the result of Davies can be applied. In this way, we obtain that there exists a uniform subset $Z$ of $\mathbf{R}^{2}$ which covers $\mathbf{R}^{2}$ by using countably many transformations from the group $G$ generated by $T_{2}$ and $g$. Therefore, $Z$ is $T_{2}$-negligible and $G$-absolutely nonmeasurable in $\mathbf{R}^{2}$. However, this method does not work for rotations of finite order.

Remark 2. We do not know whether there exists a subset of $\mathbf{R}^{2}$ which is $T_{2}$-negligible and $S_{2}$-absolutely nonmeasurable.

Now, taking into account Lemmas 2, 3 and 4, we can extend Theorem 1 to Euclidean spaces of higher dimension. For the sake of simplicity, we formulate here the corresponding result only for the three-dimensional Euclidean space $\mathbf{R}^{3}$.

Theorem 2. Let $g$ be a rotation of the space $\mathbf{R}^{3}$, whose corresponding angle differs from 0 and $\pi$, and let $G$ denote the group generated by $g$ and $T_{3}$. Then there exists a subset $Z$ of $\mathbf{R}^{3}$ which is $T_{3}$-negligible and $G$-absolutely nonmeasurable.

Proof. Let us represent $\mathbf{R}^{3}$ in the form of a direct product:

$$
\mathbf{R}^{3}=\mathbf{R}^{2} \times \mathbf{R} .
$$

Without loss of generality, we may assume that the axis of fixed points of $g$ coincides with the second factor in this product (in particular, $g \in O_{3}^{+}$). By virtue of Theorem 1, there exists a set $Y \subset \mathbf{R}^{2}$ which is $T_{2}$-negligible and $G^{\prime}$ absolutely nonmeasurable, where $G^{\prime}$ stands for the group generated by $T_{2}$ and the restriction of $g$ to the first factor in the product $\mathbf{R}^{2} \times \mathbf{R}$. Now, applying Lemma 2 to the canonical surjective group homomorphism

$$
\mathrm{pr}_{1}: \mathbf{R}^{2} \times \mathbf{R} \rightarrow \mathbf{R}^{2}
$$

we infer that the set

$$
Z=Y \times \mathbf{R} \subset \mathbf{R}^{3}
$$

is $T_{3}$-negligible in $\mathbf{R}^{3}$. Further, applying Lemma 3 to $\mathbf{R}^{2}, G^{\prime}$ and $Y$, we deduce that

$$
\cup\left\{g_{i}^{\prime}(Y): i<\omega\right\}=\mathbf{R}^{2}
$$

for some countable family $\left\{g_{i}^{\prime}: i<\omega\right\}$ of transformations from $G^{\prime}$ and, consequently,

$$
\cup\left\{g_{i}(Z): i<\omega\right\}=\mathbf{R}^{2} \times \mathbf{R}=\mathbf{R}^{3}
$$

for some countable family $\left\{g_{i}: i<\omega\right\}$ of transformations from $G$. Finally, taking into account Lemma 4 , we claim that $Z$ is $G$-absolutely nonmeasurable in $\mathbf{R}^{3}$.

Remark 3. It is not hard to verify that the group $G$ in the preceding theorem is solvable, hence amenable. Therefore, it does not admit equidecomposability paradoxes. 
Remark 4. By applying the same result of Davies, one can easily infer that there exists a subset of $\mathbf{R}^{3}$ which is $T_{3}$-negligible (or $S_{3}$-negligible) and $M_{3}$ absolutely nonmeasurable. On the other hand, there are some constructions of extremely paradoxical subsets of $\mathbf{R}^{3}$ whose existence is essentially caused by the existence of large free subgroups of the group $\mathrm{O}_{3}^{+}$(see [7] where strong versions of the Banach-Tarski paradox are discussed in detail). It would be interesting to obtain a $T_{3}$-negligible and $M_{3}$-absolutely nonmeasurable set by utilizing those constructions.

Remark 5. A proper subclass of negligible sets, consisting of the so-called absolutely negligible sets, is of special interest for the general theory of invariant (quasi-invariant) measures. Recall that these sets are defined as follows (see [4] or $[5])$.

Let $E$ be a nonempty set and let $G$ be a group of transformations of $E$ (in other words, we have a space equipped with a transformation group). Let $X$ be a subset of $E$. We say that $X$ is $G$-absolutely negligible if, for every nonzero $\sigma$-finite $G$-quasi-invariant measure $\mu$ on $E$, there exists a $G$-quasi-invariant measure $\mu^{\prime}$ on $E$ extending $\mu$ and satisfying the equality $\mu^{\prime}(X)=0$.

Note that, in general, the class of negligible sets does not form an ideal of subsets of a given space (in this connection see, e.g., [8] and [9]). At the same time, it directly follows from the definition that the class of absolutely negligible sets is an ideal in the Boolean algebra of all subsets of an original space. As mentioned earlier in Example 1, any uniform subset of $\mathbf{R}^{2}$ is $T_{2}$-negligible. On the other hand, it was shown in [10] that there are uniform subsets of $\mathbf{R}^{2}$ which are not $T_{2}$-absolutely negligible.

In connection with this fact and with Theorems 1 and 2, the following question seems to be interesting:

Let a natural number $n$ be greater than or equal to 2 . Does there exist a subset of the Euclidean space $\mathbf{R}^{n}$ which is $T_{n}$-absolutely negligible and, simultaneously, $M_{n}$-absolutely nonmeasurable? We do not know an answer to this question even in the case $n=2$. In other words, it is unknown whether there exists a subset of the plane $\mathbf{R}^{2}$ which is $T_{2}$-absolutely negligible and, simultaneously, $M_{2}$-absolutely nonmeasurable.

\section{REFERENCES}

1. W. Sierpiński, Sur un théoréme équivalent á l'hypothése du continu. Bull. Internat. Acad. Sci. Cracovie Ser. A, 1919, 1-3.

2. . R. O. Davies, Covering the plane with denumerably many curves. J. London Math. Soc. 38(1963), 433-438.

3. J. C. Simms, Sierpiński's theorem. Simon Stevin 65(1991), No. 1-2, 69-163.

4. A. B. Kharazishvili, Invariant extensions of the Lebesgue measure. (Russian) Tbilis. Gos. Univ., Tbilisi, 1983.

5. A. B. Kharazishvili, Transformation groups and invariant measures. Set-theoretical aspects. World Scientific Publishing Co., Inc., River Edge, NJ, 1998. 
6. W. Sierpiński, Sur le paradoxe de la sphère. Fund. Math. 33(1945), 235-244.

7. S. Wagon, The Banach-Tarski paradox. With a foreword by Jan Mycielski. Encyclopedia of Mathematics and its Applications, 24. Cambridge University Press, Cambridge, 1985.

8. A. B. KharaZishvili, Some questions concerning invariant extensions of Lebesgue measure. Real Anal. Exchange 20(1994/95), No. 2, 580-592.

9. A. B. Kharazishvili, Small sets in uncountable abelian groups. Acta Univ. Lodz. Folia Math. No. 7 (1995), 31-39.

10. A. B. Kharazishvili, Questions in the theory of sets and in measure theory. (Russian) Tbilis. Gos. Univ., Tbilisi, 1978.

(Received 4.09.2003)

Author's address:

I. Vekua Institute of Applied Mathematics

I. Javakhishvili Tbilisi State University

2, University St., Tbilisi 0143

Georgia

E-mail: kharaz@saba.edu.ge 